\title{
Littérature dialectale à Farciennes et Atlas linguistique de la Wallonie. De la géographie à la généricité ${ }^{1}$
}

\author{
Daniel Droixhe et Nadine Vanwelkenhuyzen
}

En 1988, on a présenté au colloque sur La formation spontanée de koinès et la standardisation en gallo-roman, tenu à l'Université de Neuchâtel, un «Coup d'œil sur l'attraction vocalique liégeoise dans la littérature wallonne de 1600 à $1850 »\left({ }^{2}\right)$. On y suivait une recommandation d'Él. Legros, qui remarquait en 1959 que « les textes littéraires en wallon n'ont pas été en général beaucoup étudiés jusqu'ici du point de vue linguistique $»\left({ }^{3}\right)$. Él. Legros mettait ainsi en évidence le rôle joué par le wallon liégeois comme pôle d'attraction régional, en examinant des textes littéraires du nord-est et de l'ouest de Liège (Herstal, Vottem d'une part, Ans de l'autre). L'exposé de 1988 considérait notamment, quant à lui, divers traits concernant la Hesbaye et la région de Verviers ${ }^{4}$.

On se propose ici d'aborder de façon plus étendue la représentation «littéraire » de traits locaux dans une autre région de Wallonie. On a choisi de s'intéresser aux écrivains de Farciennes - Ch[arleroi] 54 dans 1'Atlas linguistique de la Wallonie $\left(^{5}\right)$ en raison de la position particulière qu'occupe cette localité. Elle se situe à la frontière entre centre-wallon, ou wallon namurois (désormais nam.), et ouest-wallon (désormais o.-w.), tels qu'ils se présentent dans les diverses cartes générales des dialectes de Wallonie et dans les études plus particulières sur l'aire namuroise (J. Simon, A. Maréchal, J. Niederländer, A. Grignard, M. Valkhoff, B. Atwood, L. Remacle $)\left({ }^{6}\right)$. Notons que le recours aux textes littéraires, pour la phonétique de l'o.-w., est ancien puisque A. Grignard signale divers écarts par rapport à la prononciation " authentique ». "Bernus, Fables, remplace fréquemment an par in, surtout dans des mots qui, sans cela, seraient purement français: cominder, savint, imbicion, d'minder, rimponia, minquer », etc. Le même auteur donne viète pour le régulier vète 'verte' «par analogie » (traitement du é]) . «Les œuvres de J. Bertrand présentent quelques formes

\footnotetext{
1 Paru dans la Revue belge de philologie et d'histoire 80, 2002, pp. 875-904.

${ }^{2}$ Paru dans KNECHT (P.) et MARZYS (Z.), éd. Écriture, langues communes et normes (Neuchâtel -Genève : Fac. des Lettres de l’Univ. - Droz), 1992, pp. 133-60.

3 « Glanures linguistiques dans les textes littéraires en wallon de Liège et de Verviers », Les dialectes belgoromans, 16 (1959), pp. 5-43 et 97-126.

${ }^{4}$ Traitement des voyelles nasales selon diverses catégories (participe présent, $1{ }^{\text {ère }}$ pers. du pluriel de l'indic. présent, du futur, de l'impératif), notations de l'alphassisme verviétois, du participe passé féminin, des résultats du ú ù dans plūma et rūga, etc.

5 Atlas linguistique de la Wallonie. I. Introduction générale. Aspects phonétiques par Louis REMACLE (Liège : Vaillant-Carmanne), 1953 ; II. Aspects morphologiques par Louis REMACLE, 1969 (désormais ALW).

${ }^{6}$ SIMON (J.), « Les limites du picard et du wallon en Belgique et la question des dialectes », Mélanges wallons (Liège), 1892, pp. 99-110 ; MARÉCHAL (A.), « Carte dialectale de l'arrondissement de Namur indiquant les limites des principales variations flexionnelles des patois locaux », Bull. Soc. litt. wall. 40, (1900), pp. 65-94; ID., « La Wallonie et ses divisions linguistiques », Enquêtes du Musée de la Vie Wallonne 9-10 (1926), pp. 27383 ; J. NIEDERLÄNDER, « Die Mundart von Namur », Zeitschr. f. rom. Phil. 24 (1900), pp. 1-32 \& 251-309; A. GRIGNARD, Phonétique et morphologie de l'ouest-wallon (Liège : Vaillant-Carmanne, 1908, tir. sép.).
} 
en -èye: soulèye, pavèye, par analogie du masc. » (à la place de -éye, -îye, dans les part. passés fém.) ${ }^{7}$ ).

Farciennes ne constitue pas dans l'ALW un point d'enquête régulier $\left({ }^{8}\right)$. La forme enregistrée est parfois reportée sur carte, parfois mentionnée dans le seul commentaire, parfois absente. La localité se trouve sur la ligne verticale unissant $\mathrm{Ch} 33$ Fleurus et $\mathrm{Ch} 61$ Châtelet, ligne qui constitue tantôt la lisière de plusieurs traits namurois, ou plus largement wallons, tantôt la limite extrême de traits picards. En outre, les points Ch 33 et 61 offrent des traitements distincts, d'où le projet d'une enquête tâchant de situer Farciennes par rapport à ses voisines. En d'autres termes, Farciennes nous a paru un pt d'enquête introduisant à plusieurs niveaux de la fragmentation dialectale de la Belgique romane, selon qu'elle oppose wallon et picard ou qu'elle différencie des variétés de wallon.

On a retenu quatre auteurs, choisis parmi ceux qui écrivirent pour la scène avant la première guerre mondiale $\left({ }^{9}\right)$. Il a semblé que le dialogue théâtral - même de niveau littéraire médiocre - n'en offrait pas moins d'intérêt du point de vue linguistique. Les pièces envisagées l'ont été en fonction de leur accessibilité, soit qu'elles figurent dans les collections de la Bibliothèque des Dialectes de Wallonie à Liège (ci-dessous dénommée BDW), soit qu'elles soient conservées à la Bibliothèque royale Albert Ier (BR), qui offre à cet égard certaines richesses. C'est dire que ce choix des œuvres a été très aléatoire et que le vaudeville le moins distingué voisine ici avec la comédie digne de ce nom. Peu nous importe. Par contre, la relative authenticité de la langue constitue un critère essentiel. L'existence même de koinès dialectales rend suspecte cette notion d'authenticité, de fidélité au parler local, qui peut être natal, mais pas nécessairement. L'écrivain «d'adoption» a su également, en littérature patoise, imposer une qualité de langue apprise dans son jeune âge. L'appréciation de cette qualité est parfois rendue difficile, dans le cas qui nous occupe, par la maigreur des renseignements biographiques concernant nos auteurs. Il faut donc s'en remettre à un lieu de naissance, ou de création de telle pièce, à ce que celle-ci veut bien nous dire éventuellement de son parler, du cadre précis de l'action, de l'origine ou de la résidence des personnages, etc.

Joseph Modave est le plus connu des auteurs dramatiques de Farciennes, et apparemment le plus ancien. Il y est né en 1865 et mort à Rixensart en 1947. Après des études secondaires à Châtelet et Charleroi, il s'établit artisan-ferronnier à Farciennes, Monceau-surSambre et Marchiennes. Ayant quitté la Belgique pour le Canada et Douai, il se fixa ensuite dans la région bruxelloise, à Saint-Gilles, non sans revenir régulièrement au Pays Noir. Il y reprenait contact « avec ses nombreux amis wallons houilleurs, métallurgistes et ouvriers des champs, avec qui il converse volontiers en termes du cru », rapporte un article de 1925 dans $L^{\prime}$ hûlaud d' Châlèrwè ${ }^{10}$ ). Prolifique, il a laissé, outre chansons, poèmes et monologues, une cinquantaine de comédies, comédies-bouffes, vaudevilles, etc. Dans une conférence prononcée à l'Association littéraire wallonne de Charleroi en 1937 (texte ms. à la BDW), il évoque ses premiers essais pour la scène wallonne, en particulier $A$ l' tchesse aux mouchons, dont il sera question ici. Un article d'É. Lempereur, en 1947, le dit «pareil à lui-même :

\footnotetext{
${ }^{7}$ GRIGNARD, pp. 27 (401), 41 (415), 105 (479). Cf. E(mile) L(empereur), “A. Grignard”, El bourdon d' Châlerwè ( $36^{\mathrm{e}}$ année, ${ }^{\circ} 374$ ), 1985, 2, pp. 54-55 (avec le jugement plutôt positif d'É. Legros sur "un ensemble digne de respect", qui "a rendu et rendra encore des services malgré des erreurs et des lacunes").

${ }^{8}$ L'enquête avait été entreprise en 1936 par Jules Guyaux et vérifié par J. Haust, les témoins étant Camille Somville et Sébastienne S.-Charlier, originaire de Pont-de-Loup (Ch 53).

${ }^{9}$ Cf. VANDEREUSE (J.), Le théâtre wallon du Hainaut (Charleroi : Imp. Ed. François), 1913 (extr. de l'Annuaire de l'Ass. litt. wall. de Charleroi 3, 1912). - $2^{\text {ème }}$ éd. revue et mise à jour (La Louvière : Labor), s.d.

${ }^{10}$ Trois. année, $\mathrm{n}^{\circ} 11$, nov. ; dossier Modave à la BDW. Pour des raisons d'ordre bibliographique, on conserve ici l'orthographe originale.
} 
cordial, caustique, plein de verve, bouillant, l'imagination aussi fraîche qu'au temps des crinolines » $\left({ }^{11}\right)$. Les pièces de Modave qu'on a vues ont été imprimées à Charleroi, où l'une d'elles au moins a été créée. Elles ne comportent pas d'autres indications de localisation.

On a déjà beaucoup moins de renseignements sur Camille Malburny, né à Farciennes en 1890, mort à Marcinelle en 1936. Cet employé publia des poèmes dans les journaux

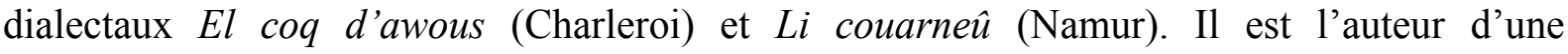
quarantaine de chansons, monologues et nouvelles, ainsi que de nombreux vaudevilles, comédies-bouffes, opérettes, etc. Son dossier à la BDW est mince $\left({ }^{12}\right)$. Ene passeye au Cafeu Napoléon se déroule dans un estaminet situé « aux environs de Fleurus », ce qui va expliquer le quiproquo constituant en principe le ressort comique de cette bambochade. Le tenancier, Napoleon Bionapôtre, profitera de la crédulité de deux touristes anglais pour leur vendre de prétendues reliques du «petit caporal». Le vaudeville fut créé à Farciennes, où l'auteur résidait au moment de la représentation. $L$ 'pêche miraculeuse y fut également créé peu après. Dédiée à Joseph Modave, la pièce a pour cadre l' "auberge de la Bredouille ", tenue par Isidore Rapêchî («Repêché »), organisateur d'un concours de pêche à la ligne qui " aura lieu à Farciennes, dans la rivière dénommée "la Sambre" ». Le réalisme de la situation et du décor plaide en principe pour un tableau de mœurs locales offrant un certain caractère de vérité linguistique. L'action de Quéle affaire! se passe aussi - comme dans tant d'autres pièces du répertoire carolorégien - dans une auberge implicitement située à Farciennes : le personnage de Constant y revient incognito pour accomplir une vengeance, après avoir quitté la localité « voilà deux ans ».

On dispose de moins d'informations encore concernant Ernest Guyaux, né à Farciennes en 1888 (pas de dossier à la BDW). Sa comédie Savèz bén qu'hier fut publiée dans cette localité en 1929. Il a également écrit, pour la scène, en collaboration avec Firmin Callaert, originaire de Dampremy : la localité se trouvant à l'ouest de Ch 1, sur la ligne de Ch 43 Jumet, il y aurait peut-être à considérer comment, malgré la courte distance les séparant, les deux auteurs ont résolu la question des différences de parler local.

Il n’y a pas de dossier, à la BDW, sur Camille Renson. Sa comédie des Deux galants da Eva se présente comme écrite en patoët d' Farciennes.

Notre étude ne prétend donc en rien exploiter toute la liste des auteurs dramatiques du cru, même ceux ayant écrit avant 1914. On n'a pas trouvé à la BDW et à la BR les vaudevilles de Henri Vandendries, né à Rillaer en 1876, censé écrire, d'après Vandereuse, dans le dialecte de Farciennes, pas plus que la comédie-vaudeville One pasquée on djô̂ d' novel an de François Dury, originaire de Farciennes.

La liste des ouvrages consultés, dont on reproduit des extraits dans l'orthographe originale, accompagnés du signe ${ }^{\circ}$, s'établit comme suit :

Gs Guyaux, Ernest. Savèz bén qu'hier..., Farciennes, 1929.

MAn Malburny, Camille. Ene passeye au Cafeu Napoléon. Vaudeville en un acte, Namur, J.B. Collard, 1911 [éd. à laquelle on renvoie ici ; BDW, Société de Langue et de

\footnotetext{
${ }^{11}$ Journal de Charleroi, 14 juill. 1947.

${ }^{12}$ Capable des monologues les moins délicats (In cas d' rupture !, Occupet), Malburny touche davantage, même si l'art reste court, dans un poème comme Douce erlique, où il évoque la vieille «bwèsse à ch'nouf » (boîte de tabac) de son "parain Nèsse », et à travers elle les veillées bercées par la lecture de Barbe-bleue et du Petit Poucet (voir la Nouvelle gazette du 10 juin 1986).
} 
Littérature wallonnes, désormais SLW] - Enne passeye au Cafeu Napoléon, $2^{\mathrm{e}}$ éd., Peetermans-Scory, 1913 . " Joué pour la première fois à Farciennes, le 30 avril 1911, en la soirée dramatique de la "Gymnastique Farciennoise" ".

MAp Malburny, Camille, L' pêche miraculeuse. Vaud'ville en 1 acte avec chants. Cette pièce a été créée fin 1911 à Farciennes, Charleroi, Impr. du Tonnia d'Charlerwet, 1911. [BDW, SLW]

MAq Malburny, Camille, Quéle affaire! Ou l'vengeance d'in bârbi d'occasion. Comédiebouffe en 1 acte, Charleroi, Impr. du Tonnia d' Charlerwet, 1912. [BDW, SLW]

MOf Modave, Joseph, Li fie dou marchau, Charleroi, Aux 100.000 Chansons.

MOm Modave, Joseph, On marie Fonfonse. Opérette bouffe en in aque, Charleroi, Aux 100.000 Chansons, s.d.

MOs Modave, Joseph, Au strin dzou l'huche. Vaudeville en in aque, Charleroi, Aux 100.000 Chansons. Musique et librairie théâtrale. Pasque et Christiaens Sœurs. 5, passage de la Bourse.

MOt Modave, Joseph, A l'tchesse aux mouchons. Vaud'ville en in ake. Pièce couronnée au concours de 1896 organisé par le club "Les Wallons de Liège ". Créée par le "Cercle wallon de Charleroi » à l'Eden-Théâtre le 12 mars 1898, Paris, Passage Brady, 92 - Charleroi, Aux 100.000 Chansons. [BDW, SLW]

$\mathrm{Rg} \quad$ Camille Renson, Les deux galants da Eva. Comédie en 1 acte avec chants. Patoët d'Farciennes, Charleroi, Impr. du Tonnia d' Charlerwet, 1910. [BDW, SLW]

On comprend que l'exercice comparatif qui suit offre un double projet: a) compléter, à propos d'un pt d'enquête occasionnel, les informations suggérées par l'ALW et proposer à notre tour un état probable ou possible des lignes d'isoglosse passant dans la région de Fleurus-Châtelet ; b) apprécier le degré d'authenticité de la langue des auteurs considérés. On a fondé la comparaison sur les deux premiers volumes de l'ALW (Aspects phonétiques et morphologiques). On reproduit, avec l'aimable autorisation de la direction de l'ALW, des extraits de ses cartes, dont les légendes ont été aménagées pour des raisons de place. On a, dans un certain nombre d'entre elles, reporté au pt Ch 54 le résultat de l'examen des textes (cartes avec addition).

\section{A. LA LIGNE FLEURUS-CHÂTELÊT COMME FRONTIÈRE NAMUROISE}

\section{A.1. La ligne Fleurus-Châtelet en lisière d'une dipht. nam. wa dans ÉTOILE et POIRE}

Ad. Grignard écrit que é $[$, « passant par $e i, o i, o e ́$, aboutit en général à wè » dans l'o.w. et particulièrement dans la rég. de Charleroi : "crwè (crēdo), crwère (crēdere); trwè, twè (trēs); swè (s̆̌tem); qwè (qul̆d); dwè (dēbeo); vwè, wè (vŭdeo); mwè (mēsem); pwère (pŭram); avwè (à Ittre) et awè (habēre); savwè et sawè (sapēre); stwèle $\left(\operatorname{stēlla)»,~etc.~}{ }^{13}\right.$ Niederländer fournit des formes analogues pour $\mathrm{Na}$, dans la majorité des cas. Grignard peut ainsi, à propos du cas particulier que constitue le traitement du é $[+\mathrm{y}$ (dirēctum), dessiner autour de Ch 1 et vers Namur une vaste zone homogène en wè (carte V; reprod. 22).

Le traitement du é [ est illustré de manière générale dans les vol. 1 et 2 de l'ALW, par les c. 38 ÉTOILE, 75 POIRE, 91 SOIF, 100 (IL) VOIT, 81 VOULOIR, 82 AVOIR (reprod. 1-6). La concordance avec Grignard et Niederländer n'est pas complète. En effet, un type nam. en wâ,

\footnotetext{
${ }^{13}$ GRIGNARD, p. 36 (410). On ne retient ici que les termes qui nous concernent.
} 
$w a$ affecterait les t. ÉTOILE et POIRE, et s'étendrait, en l'englobant, jusqu'à la ligne Ch 33-61. L'ALW enregistre pwâre 'poire' à Farc., alors que Grignard note pwère et stwèle. Par contre, l'ALW atteste bien la conformité des termes SOIF, VOIT, VOULOIR, AVOIR au type en wè : il donne wèt '(il) voit' pour Farc. et voulwêr 'vouloir' et awè 'avoir' pour Ch 33-61 $\left({ }^{14}\right)$. Niederländer est dans l'ensemble d'accord avec Grignard, bien qu'il note aussi à Na stwale, considéré comme un cas particulier, ainsi que les nam. bwâre, - $a$ - 'boire', tonwâr, - $a$ 'tonnerre', etc. - t. où le changement de timbre a pu être lié au $-r$ final, à moins qu'ait joué dans le premier cas l'influence fr. On peut dès lors se demander si pwâre ne constitue pas aussi un cas particulier ayant subi la double infl. du fr. et de la finale La discordance entre les notations de Grignard/Niederländer et l'ALW sont frappantes, car celui-ci dessine une vaste zone homogène avec pwâre, - $a$ - s'étendant à partir de Namur sur les arr. de Philippeville, Dinant et Neuchâteau.

Le témoignage des textes ne portera bien sûr que sur la situation à Farciennes. Ils adoptent massivement et dans tous les cas le type wê, wè de l'o.-w. Ceci vaut d'abord pour les mots enregistrés sous cette forme dans l'ALW : ' voèt, voès '(il) voit' (Gs 14, MOf 10, MOm 3, MOs 3, Rg 4), 'arr'wèr 'au revoir' (Gs 12), 'awet 'avoir' (Gs 18, 19), 'sawè 'savoir'(Gs 19) $\left({ }^{15}\right)$. Les verbes en -ēre se conforment au modèle awèt 'avoir' / wèt '(il) voit' : ${ }^{\circ}$ boère, boëre, bwès, - $t$, 'boire, (je, il) bois, -t'; ${ }^{\circ}$ crwère, croère, crwè(s), croès 'croire, (je) crois' (Gs 18, 21, 22, MAn 4, 24, MAq 8, 11, MOf 6, MOm 2, 7, 12, MOs 2, Rg 4), dwès, -t, doès, doët '(je, il) dois, -t (Gs 6, 14, 24, MAq 7, MOm 2, 14, $\operatorname{Rg} 4)\left({ }^{16}\right)$. Les t. suivants observent la même règle : ${ }^{\circ}$ foëres 'foires' $(\operatorname{Rg} 6)$, 'moès, moës 'mois' (Gs 11, MOs 2, Rg 4), 'trwès 'trois' (MOf 12, MOm 2), etc.

Le type en wâ, - $a$ - apparaîtrait-il dans les graphies ${ }^{\circ}$ soie 'soif' (MAn 9, 12) et ${ }^{\circ}$ stoile,

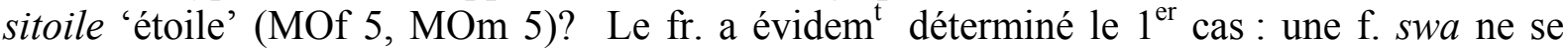
manifeste sur la c. SOIF que très loin de Farc. (ici aux frontières du lorrain, du chestrolais et du champenois ; reprod. 3). Les f. ${ }^{\circ}$ stoile. et ${ }^{\circ}$ sitoile ont pu subir la même influence, la seconde intervenant en outre dans le discours plaisant d'un nigaud (effet comique ?).

En conclusion, il semble donc que les c. ÉTOILE et POIRE ne soient pas représentatives du traitement général du é[ et même qu'elles induisent en erreur en suggérant par extrapolation pour Farc. - et p.-ê. pour la ligne Fleurus-Châtelet - une appartenance à un type $w \hat{a}, w a$. Globalement, les textes demeurent conformes au type wè noté par Grignard (à une date plus ancienne ? chez des témoins plus âgés ?) et respectent l'unité de la zone que définit sa carte V (reprod. 22).

On note chez Guyaux l'effet d'une tendance qui affecte sporadiquement l'ensemble du domaine belgo-picard et l'arrondissement de Nivelles (v. les c. 24 CRAIE et 45 FROID) : l'amuissement du $-r$-dans ${ }^{\circ}$ cwère, cwès 'croire, (je) crois' $(\mathrm{s} 14,20),{ }^{\circ} d$ wète 'droite' (à côté de ${ }^{\circ}$ drwète ; s 9, 12). Dans la c. FROID, l'aire d'extension du phénomène, qui se produit aussi devant voyelle d'arrière (fwod), s'arrête au nord de Ch 43 Jumet et Fleurus. Le parachutage de la contamination à Farc. pourrait donc étonner, si la dispersion et le relatif isolement des pts

\footnotetext{
${ }^{14}$ Bien que se manifeste à Ch 61 Châtelet la tendance nam. à substituer par analogie le part. passé à l'inf. dans le cas de VOULOIR : voulu - tendance qui affecte également « sur des aires très variables » les v. en -ere comme POUVOIR, VALOIR, FALLOIR, DEVOIR, SAVOIR, AVOIR (ALW 2, 224, $\alpha$ ).

${ }^{15}$ Le signe ${ }^{\circ}$ indique qu'il s'agit d'une f. telle qu'orthographiée dans les textes; il s'applique à une série de $\mathrm{f}$. successives.

${ }^{16}$ Et aussi : boërai, boèrons, bwérons (je) boirai, (nous) boirons' (MAn 4, 9, 12, 18bis, 23, 25, MAp 10, MOm 24).
} 
d'occurrence de l'amuissement n'étaient de nature à faire croire à des survivances ou archaïsmes, confrontés à l'expansion de f. dominantes avec $f r$-, $c r-$, etc., plus proches du français.

\section{A.2. La ligne Fleurus-Châtelet en lisière de la finale nam. - euwe $<-\bar{u}$ ta}

La c. 72 PERDUE de l'ALW 1 oppose le nam. -euwe, dont la ligne forme l'avancée extrême, et la finale -uwe à l'o., de Charleroi à Mons, Ath, etc. (reprod. 7). Les qqs f. recueillies dans les textes confirment l'ALW: 'èdoirmeuwe 'endormie' (MOs 2), 'distindeuwe 'éteinte' (MOs 8), 'div'neuwe 'devenue' (Rg 4), 'tindeuwes 'teintes' (MOm 14). On notera le traitement du pt chez Grignard, qui note pour l'o.-w. pièrduwe, èrçuwe, tandis que Maréchal, plus correctement, évoque la finale -eùwe pour Fleurus $\left({ }^{17}\right)$.

\section{A.3. La ligne Fleurus-Châtelet en frontière partielle du nam. -oz dans (VOUS) ÊTES et (VOUS) AVEZ}

Grignard fournit une carte très précise des systèmes en présence dans l'o.-W. en ce qui concerne la $2^{\text {ème }}$ pers. du plur. de l'ind. prés. (c. III). Ceux-ci se divisent d'abord en deux classes, selon qu'on considère les trois conjugaisons et leur finale respective (-atis, iatis / -yatis, -ētis). Des systèmes homogènes en -èz, au s.-o, et en -oz, au s.-e., s'opposent à des systèmes partiellement hétérogènes, combinant les types $-\dot{e} z$ et $-\hat{\imath} z$, ou totalement hétérogènes, combinant les types $-\dot{e} z,-\hat{l} z$ et $-o z$. La ligne Fleurus-Châtelet se trouve localisée dans l'aire régie par ce dernier système et la "limite d'une zone où les systèmes se confondent » passe à peu près par Farciennes.

On se bornera ici à l'examen des v. être et avoir, qui appartiennent à la $3^{\text {ème }}$ conj. Selon la c. 103 ÊTES de l'ALW 2, Farc. et Fleurus relèvent d'une zone nord/namuroise où domine le type en $-O z$ (èstoz), tandis que Châtelet a un type en - ̀̀z (èstèz) qui s'étend sur Charleroi et Philippeville (v. reprod. 8) $\left({ }^{18}\right.$ ). Le type nord/nam. gagne Châtelet dans la c. 102 (VOUS) AVEZ (reprod. 9) : y coexistent avèz et avouz (situation identique dans la c. 100 VENEZ).

Les textes reflètent l'hésitation d'une «zone où les systèmes se confondent », mais les plus fiables d'entre eux confirment l'appartenance de Farc. à la zone nord/nam. Nos auteurs, en particulier Modave, ont unanimement le type $-o z$ : ' estos '(vous) êtes' (MOf 8, MOt 9, Rg 5), 'avoz, -os '(vous) avez' (MAn 4, 5, MOf 3) - de même ${ }^{\circ}$ savos, -o 'savez ' (MOf 10, MOs 3), 'plos 'pouvez' (MOf 16), 'v'nos 'venez' (MOf 9), 'fios 'faites' (Rg 8), etc. Il est vrai que Guyaux, p.-ê. moins crédible, a des f. du sud : ${ }^{\circ}$ estèz (s 17), ${ }^{\circ}$ avèz (s 16, 19, 22) - de même ${ }^{\circ} v l$ 'èz 'voulez' (s 14, 17, 19), 'allèz (s 18).

Alph. Maréchal notait par ailleurs : « À Namur et aux environs, dans la tournure interrogative, le pronom sujet vò manque toujours : ki dj'ò ? compurdò ? (que dites-vous ? comprenez-vous ?). Ailleurs, le pronom a laissé des traces : c'est le long de la frontière du Hainaut (...), où l'-ò- de flexion devient -ó- en cas d'interrogation : astó contint ? avó dîné ? Cet ó provient de ò + vò...» $\left({ }^{19}\right)$. Parmi les localités manifestant le trait, Maréchal mentionnait Fleurus et Farc., ce que l'ALW 2, c. 101 VOULEZ(-VOUS) confirme pour Farc. : volôz. Nos textes l'enregistrent également : 'avô 'avez-vous' (MOf 6, 8, MOm 16), 'savô 'savez-vous' (MAn 7, MOs 11, MOf 10), 'pinsô 'pensez-vous' (MOf 6), 'intrô 'entrez-vous'

\footnotetext{
${ }^{17}$ MARÉCHAL 1900, p. 81 ; GRIGNARD, p. 56 (430).

${ }^{18}$ La distinction - èz -oz sépare aussi Fleur. et Chât. dans un contexte différent sur la c. 97 LEVEZ de l'ALW 2.

${ }^{19}$ MARÉCHAL 1900, p. 75.
} 
(MOf 10), 'cachô 'cherchez-vous' (MOm 16). Renson se singularise ici par des f. en -ou caractéristiques de Châtelet : 'avou 'avez-vous' ( $\operatorname{Rg} 7)$, 'savou 'savez-vous' ( $\operatorname{Rg} 5$ ), 'n'estou né èvoye à l' dicausse di Pont-d'Loup? 'n'êtes-vous pas parti à la ducasse de Pont-deLoup ?' (Rg 11).

Farc. appartient bien au dom. nam. $\left({ }^{20}\right)$. La localité subit dans une certaine mesure, chez Guyaux et Renson, l'attraction des deux types en concurrence à Châtelet, avec finales en -èz (pic.) et - ouz (nam.).

\section{A.4. Farciennes en lisière du nam. $-r o z$, indic. futur, $2^{\text {ème }}$ pers. du plur.}

Farc. occupe une position très particulière selon la c. 107 (VOUS) AUREZ de l'ALW 2 (reprod. 10), puisque Ch 54 constitue un pt avancé du type nam. en -roz, auquel s'opposent à l'e. et à l'o. les types liégeois et o.-w. en -réz, -rèz. Le plus fidèle de nos auteurs, Modave, a le type nam. : 's'ros 'serez' f 24 ; cf. Rg 5), 'pôro 'pourrez' (f 25), 'trouveros 'trouverez' (MOf 15), etc. Mais Renson hésite : ${ }^{\circ} s$ 'ros 'serez' (Rg 5), 'sèret 'serez' (Rg 14).

\section{B. LA LIGNE FLEURUS-CHÂTELÊT COMME FRONTIÈRE PICARDE}

\section{B.1. La ligne Fleurus-Châtelet en lisière du système pic. des 3 pers. du sing. aux indicatifs présent et futur}

La position particulière qu'occupent ici la ligne Ch 33-61 et Farciennes selon l'ALW doit être envisagée globalement, pour le prés. et fut. La c. 92 (J')AI place cette ligne en lisière du type pic. é, opposé au wallon $a$ (reprod. 11). La position de Farc. est plus remarquable encore dans les c. 105 (JE) DIRAI et 106 (IL) SERA, où la localité est désignée comme pt extrême du domaine pic., avec des fin. en $-r e ́\left(1^{\text {ère }}\right.$ pers. $=$ fr. $\left.-r a i\right)$ et $-r a\left(3^{\text {ème }}\right.$ pers. $\left.){ }^{21}\right)$ tandis que Ch 33 Fleurus a le type wallon unitaire en -rè (reprod. 12-13). Maréchal soulignait déjà une différence générale entre un régime w. où les 3 pers. du sing. au présent et au futur sont homogènes (type lg. $d j$ 'a, t'as, il a // dj'årè, t' årès, il årè) et un régime pic. où les f. sont hétérogènes (type ai, as, a // aurai, auras, aura) $\left({ }^{22}\right.$ ). Il y ajoutait un troisième régime, mixte, mitoyen, qui présente une flexion non-uniforme au prés. et uniforme au fut.: situation illustrée, comme on le voit ci-dessus, par Ch 33 Fleurus. Maréchal constatait donc que le système wallon unitaire («monotone» dit-il) se démembre notamment vers Farciennes. Grignard propose le tracé d'une ligne unique de séparation entre les deux régimes principaux - méconnaissant le fait que les frontières entre pic. et w., pour le prés. et le fut., ne peuvent se superposer (c. XI ; v. reprod. 14) $\left({ }^{23}\right)$.

Apprécier la fidélité des textes à ces données s'avère évidemment délicat, en raison de la pression exercée par l'orthographe française. Notera-t-on l'absolue conformité des graphies au système pic. de l'ind. présent? On trouve : ${ }^{\circ} d j$ 'ai (Gs 14, MAp 3, MAq 9, MOt 3), ${ }^{\circ} d j$ ' n'ai, ${ }^{\circ}$ dji n'ai (Gs 11, MOf 7, Rg 11). Le cas du fut. est compliqué par l'éventuel risque de

\footnotetext{
${ }^{20} \mathrm{Ce}$ qu'indiquait le texte de Grignard, qui note une tendance à l'uniformisation en - $O z$ (la c. III est moins claire).

${ }^{21}$ Indication sur carte, non reprise en commentaire (A.II), mais mentionnée en note 1 dans les « autres q. consultées » : Ch '54 sốkèlrè́.

${ }^{22}$ MARÉCHAL 1900, pp. 85-86. On sait que pour L. Remacle, le système w. du futur, procédant par extension analogique de la flexion de la $1^{\text {ère }}$ pers. aux deux autres, se différenciait dès la fin du XIIIe siècle par rapport aux systèmes pic. et gaumais.

${ }^{23}$ Celle-ci, allant de La Hulpe (Ni 10) et Ohain (Ni 39) à l'o. de Ph 81 Vierves, passe tout près de Fleurus, Farc. et Châtelet, qu'elle laisse en domaine pic.
} 
confusion que présentent des finales en $-a i$, susceptibles de reproduire le pic. $-e ́$ ou le $-\grave{e}$ du wallon : 'aurai, arai 'aurai' (Gs 11, MOt 3, MOm 2), ${ }^{\circ}$ srai, s'rai 'serai' (MOs 7, Rg 4), 'pourrai (Gs 12, 22), 'boërai 'boirai' (MAn 3), etc. On peut néanmoins croire ces graphies conformes au type pic., dans la mesure où -è se trouve régulièrement rendu par ${ }^{\circ}$-ais et où la f. 'rindré authentifie le timbre (Gs 16). Dans le même sens, les f. de la $3^{\text {eme }}$ pers., au fut., suivent le système pic. : ' $s$ 'ra 'sera' (Gs 11, Rg 4), 'ça t'apurdra 'ça t'apprendra' (Gs 11), 'ti n'èl conira 'tu ne le connaîtras' (Gs 11), 'Faura bé printe li timps d' moru 'Faudra bien prendre le temps de mourir' (MOf 8), etc.

Est-ce à dire que nulle influence ou attraction du w. n'est perceptible dans les textes ? Malburny a qqs graphies en - $\dot{e}$ : ' sèrait 'sera' (MAn 3), 'frais 'fera' ( $n$ 15).

La f. ${ }^{\circ}$ dj'ira pose problème. On croirait à une extension analogique des f. pic. de la $2^{\text {ème }}$ et $3^{\text {ème }}$ pers., avec uniformisation de type $\mathrm{w}$., si ${ }^{\circ} d j$ 'ira ne rimait, dans la chanson où la $\mathrm{f}$. apparaît (MOf 24), avec ${ }^{\circ} \mathrm{cwai}$ 'chercher', qui ne peut guère se lire que cwé (cf. qué dans le Dict. de l'ouest-w. de Carlier) $\left({ }^{24}\right)$ ou cwè. Dans ce dernier cas, le w. en -è de la $1^{\text {ère }}$ pers. du fut. a pu exercer une attraction cumulée avec celle, plus structurelle, de l'uniformité.

\section{B.2. La ligne Fleurus-Châtelet en lisière du picard -iêre <-aria}

La c. 79 POUSSIÈRE de l'ALW 1 oppose le type -êre à Na et dans une partie du Brabant w. au type pic. en -iêre (reprod. 15). La ligne Fleurus-Châtelet fournirait la frontière du domaine pic. Pour Grignard, le type pic. aurait évincé, avec l'aide du français, la f. nam./w. Ceci semble vérifié dans nos textes par des f. comme ${ }^{\circ}$ poûssiêre (MAp 9), mais des t. également soumis à l'influence fr., comme première, connaissent également une forme « difficilior»-plus authentique : 'premère 'première' (Gs 3, 20). Voir aussi 'fumère 'fumée' (MOs $3 ;<*$ fumaria) $\left({ }^{25}\right)$. Farc. semble donc appartenir au domaine nam., ce qui suggère de reconsidérer la situation de la ligne Fleurus-Châtelet, le type en -êre ayant pu s'étendre de cette ligne jusqu'à $\mathrm{Ch} 72$ Gerpinnes. On note à nouveau que les textes semblent enregistrer des f. plus anciennes que celles relevées pour l'ALW, comme dans le cas du é[ (ÉTOILE, etc.).

\section{LES VARIATIONS DU $O$ LATIN : LA LIGNE FLEURUS-CHÂTELET EN FRONTIÈRE W. OU PIC.}

\section{C.1. Fleurus-Châtelet en zone frontière du wall. ou, ô $<$ ŏ́[}

La c. 4 BEUF de l'ALW 1 oppose une aire proprem $^{t}$ w. en $o u$, ô à une aire pic. avec $i e u$, eu, êu, $\hat{u}$, wè, etc. Le triangle constitué par Ch 43 Jumet, Ch 33 et Ch 61 forme l'avancée extrême du type w. (résultant d'une monophtongaison de la diphtongue lat. úǫ qui se développe en Gaule, en w. comme en fr., du IIe au IVe s.) $\left({ }^{26}\right)$. Rien d'étonnant à ce que nos

\footnotetext{
${ }^{24}$ Publié sous la direction de W. Bal (Charleroi : Ed. de l'Association royale littéraire wallonne de Charleroi), $1985 \mathrm{sv}$.

${ }^{25}$ Rien à tirer, par contre, de la f. ${ }^{\circ}$ plandjère 'sieste après le repas de midi, méridienne' (MAq 6 ; < *prandiaria), citée par GRIGNARD, p. 25 (399).

${ }^{26}$ ZINK (G.), Phonétique historique du français (Paris : PUF), 1986, pp. 50-55. Cette diphtongue produite lors d'une première phase de différenciation, comme celle affectant à la même époque le ě lat. > íę, se réduit en w. par résorption du second élément dans le premier, constitué de la voy. vélaire ou palatale la plus fermée (L. REMACLE). Si la chronologie w. des changements peut se référer à celle du fr., cette réduction aurait dû avoir lieu avant la seconde phase de différenciation, qui intervient en fr. aux XIe-XIIe s. et qui remédie au rapprochement de úǫ > ụ́ (v. VIIe s. ?) par palatalisation de u $>$ ü et labialisation de ọ $>$ @e d'où le fr. cüœr
} 
textes aient des graphies concordantes : ${ }^{\circ}$ plout '(il) pleut' (MAp $4 ;<*$ plŏvit), ${ }^{\circ}$ pou, $-t$ '(je, il) peux, -t' (Rs 11, 16, MAn 4), ${ }^{\circ}$ voux, -t '(je, il) veux, -t' (Gs 11, MAp 5), en alternance avec ${ }^{\circ}$ voûx '(je, tu) veux' (MAp 9, 10), etc. (v. reprod. 17). On notera cependant que le commentaire de l'ALW donne pour Farc.des formes longues, sans reporter l'information sur la carte.

\section{C.2. Fleurus-Châtelet en lisière du wall. $w a, w \hat{a}<\breve{o}]+\mathbf{r}$}

La diphtongaison de $\breve{o}]+-\mathrm{r}$ ou $-\mathrm{s}$ constitue un des «facteurs les plus nets de segmentation » du domaine belgo-roman (L. Remacle). Il caractérise fortement le w. par opposition au pic. et au gaumais/lorrain, qui conservent la voy. simple. La datation du phénomène par rapport à la diphtongaison du ŏ́ [e bŏvem est difficile à déterminer. Mais les Gloses de Darmstadt permettraient d'avancer que le namurois, qui nous intéresse ici, avait atteint au XIIIe s. - c.-à-d. à l'époque où pwère 'poire' devient pware à Paris - le terme wa, après être passé par le stade wè du liégeois. Le domaine du type $w a$, wâ est défini dans l'ALW 1 par les c. 62 MORT, 63 MORTE et 77 PORTER, que l'ŏ] soit tonique (MORT, MORTE) ou en position atone (PORTER). L'aire de diphtongaison est celle du centre-w., couvrant Nivelles-e., Namur et l'ardennais. La ligne Fleurus-Châtelet en constitue l'avancée extrême. La zone sans diphtong. commence à l'o. avec Ch 43 Jumet, qui a môrt, môrte, pôrter, et s'étend en direction du pic. sur le reste de l'arr. de Ch, l'o. de Ni, Ph et Thuin, c.-à-d. l'o.-w. L'ALW enregistre les f. w. mwârt et mwate à Farc. $\left({ }^{27}\right)$. Grignard fournit deux cartes (VI et XII) montrant en effet que la frontière passe quasi verticalement à l'o. de Châtelet, qui est donc en domaine w. (voir reprod. 18-20).

Les mots concernés se conforment généralement, sans ambiguïté, à ces indications prises sur le terrain, que la voy. lat. de l'étymon soit tonique ou non : ${ }^{\circ}$ mwoirt, moirt 'mort' (Gs 11, MOs 2), 'pwoite, poite, pwoirter, poirtons, poirté, apoirter, rapoirtant '(je) porte, porter, portons, porté, apporter, rapportant' (Gs 3, 8, 14 ; MOf 5, 25, MOm 22, MOs 2 ; MAn 14), 'doit, dwoit, (s')èdwoit, dwarmu, dwoirmu, doimerai '(je) dors, (il s')endort, dormir, dormirai' (Gs 8, 14, MAq 21, MOm 7, 12 ; < * dŏrmo), ' fwart 'fort' (MAq 5, 6, MOf 14 , 20, MOm $12, \operatorname{Rg} 6,10),{ }^{\circ}$ coine ‘corne' (MOs 5), ' coittes ‘cordes' (Rg 9), etc.

Nos auteurs, cependant, laissent échapper l'une ou l'autre forme monophtonguée : 'tôrt 'tort' (MOf 25), 'suppaurte 'supporte' (Rg 9). Ces écarts sont plus nombreux chez Malburny: 'èpaurter 'emporter' (n 20), 'daurmi, -mu, -mais, èsdaurmi 'dormir, -mais, endormi' (q 6, 12, 17), `faurt, fôrte 'fort, -te' (n 3, 5), 'amoûces 'amorces' (p 15). Malburny peut faire dire à un même personnage et dans un même passage l'hybride ${ }^{\circ}$ Dji daurmais fwart 'Je dormais fort' et peu après ${ }^{\circ}$ Ça n' mi paraît né faurt clair 'Ça ne me paraît pas fort clair' (q 18-19). Les exemples de ${ }^{\circ}$ suppaurte et 'èpaurter suggèrent que les termess composés conservent moins bien le type original et sont davantage soumis à l'infl. du fr. ou de parlers voisins.

\section{C.3. Fleurus-Châtelet en lisière du pic. ou $<\overline{0}]$}

\footnotetext{
'cœur' (ZINK, p. 55). De là l'attestation, en 1179, de la monophtongaison w., p.-ê. nettement plus ancienne. GRIGNARD mentionne pour le domaine nam. qqs f. n'obéissant pas à l'évolution en ou, ôu : mort <*mŏrit, fors < fŏris, sieur/cheur < sŏror, keûr < cŏrem, neuf < nŏvem, certaines de ces f. s'expliquant par l'infl. du fr. ; NIEDERLÄNDER exceptait aussi rêuwe < rŏta (cf. ALW 1, c. 85 ROUE).

${ }^{27}$ La f. fém. manque à la carte.
} 
La c. 25 CROÛTE oppose une zone proprem ${ }^{t}$ w. en $Q$ à une zone en ou constituée du pic. et de 1'o.-w., dont la ligne Ch 33-61 constitue la lisière. Les f. de Farc. suivent ce second régime: ${ }^{\circ}$ couches 'branches'(MOm 19: ' fallait qu'on s' teigne aux couches, $\mathrm{cf}$. $s$ ' tèni a lès couches 'se cramponner, tenir bon, résister', d'après Carlier ; < lat. cōxa), 'crousse 'croûte' (MOf 12), 'goute, gouttes 'goutte(s)' (Gs 11, MOs 2), 'tout 'tout' (Gs 17, MOm 20 ; < lat. vulg. de Gaule *tōttu), etc. (reprod. 21). Grignard détaille les réalisations avec ou dans l'o.w., tandis que Niederländer met l'accent sur des disparités namuroises étrangères à notre propos $\left({ }^{28}\right)$.

\section{Formes en concurrence}

\section{D.1. Formes en concurrence entre Fleurus et Châtelet}

\section{D.1.1. Fleurus wè̀, $\grave{e} \sim$ Châtelet $e \hat{u}<\overline{\mathbf{e}}+\mathbf{y}$}

La c. V de Grignard et la c. 45 FROID opposent un type général nam. -wè-, frèd, au n. de Farc., à un type -êै-, freud au s., qui couvre $\mathrm{Ph}$ (reprod. 22-23). Les auteurs ont général ${ }^{\mathrm{t}}$ ${ }^{\circ}$ droëte, drwète 'droite' (Gs 12, MAq 11, MOm 2), 'dwèt, doèt 'doigt' (Gs 3, 4, MOm 12, Rg 5), 'rwèt, roèt 'roide' (MAq 10: 'pu rwet qu' bîche 'plus roide que bise'; MOs 6: 'tes affaires ni vont né roèt 'tes affaires ne vont pas fort', Rg 40). Le terme FROID choisi par l'ALW constitue un cas quelque peu particulier, en raison de l'amuissement du $-w$ (allègement du groupe tri-consonantique) : ${ }^{\circ}$ frèd , frêd (MAn 8bis, 14, 15). Plus détaillé que la carte qui situe la frontière des deux types entre Fleurus et Châtelet, le commentaire de 1'ALW fait passer celle-ci par Farciennes. Pour Grignard, elle descend jusqu'à Ch 72 Gerpinnes.

On a évoqué (A1) l'amuissement du $-r$ - , de type pic. et nivellois, qui affecte drwète $>$ ${ }^{\circ} d$ wète et ${ }^{\circ}$ rwèd 'roide' $>{ }^{\circ}$ wèt chez certains auteurs. On conçoit que frwèd 'froid', pour remédier à l'accumulation consonantique, n'applique pas, dans certains cas, une réduction risquant d'entraîner une confusion homonymique avec fwète 'foie', relevé par Grignard (37) sous la f. fwè à Th ' 35 Nalinnes/Ham-s.-Heure ( $<\star$ fécatum avec métathèse des cons. $c$ et $t$ $>$ *éticum : v. Wartburg). D'où p.-ê. la réduction en frèd, du même type que celle qui affecte crèche 'croître' (< crēscere) relevé par Niederländer pour Na.

\section{D.1.2. Fleurus $\tilde{\boldsymbol{a}} \sim$ Châtelet $\tilde{\boldsymbol{o}}<\mathbf{a ]}+\mathbf{n}, \mathbf{m}$}

Si la c. 58 MANCHE offre uniformément la voy. nas. an pour la ligne Ch 33-61, la différence signalée en titre apparaît dans les c. 9 CHAMBRE et 52 JAMBE (reprod. 24). Châtel. vélarise ici en tchåmbe, djåmbe, c.-à-d. en des f. proches de tchombe, djombe. Nos auteurs ont le type $\tilde{a}$, à l'exception de Malburny: 'divont 'devant' (n 24), 'dondji 'besoin' (q 14), ${ }^{\circ}$ dondjureu littér. 'dangereux', adv. 'probablement' (n 15, p 5 ; à côté de ${ }^{\circ}$ dandjureux n 6), 'donser 'danser' ( $\mathrm{p} 5)$, 'grond, -de (n 10, 17, p 4, 16), monqué 'manqué' (p 11), 'quond 'quand' (p 4, 5), 'tont 'tant' (q 8).

\footnotetext{
${ }^{28}$ NIEDERLÄNDER opposait principalement les réalisations nam. du ō/ŭ] ton. en $o$ et en $o \hat{u}: \cos$ ' 'coûte' < cōnstat, dope 'double' < dŭplum, fôr 'four' < fŭrnu, fotche 'fourche' < fŭrca, tos' 'toux' < tŭssem, etc. boûsse 'bourse' < bōrsa, foûme 'forme' < fōrma, poûre 'poudre' < pŭlverem, toûne '(il) tourne' < tōrnat, etc. Certaines f. nam. en ou/oû ont dû subir l'infl. du fr. : bouche < bŭcca (lg. boke), mouche < mŭsca (lg. mohe), coûrs '(je) cours' < cŭrro. D'autres, que le lg. partage avec le nam., montreraient une vocalisation du $l$ entravant, comme poûre < pŭlverem ou choûte/ascô̂te '(j')écoute' < auscŭlto.
} 
D.1.3. Fleurus $\tilde{e} \sim$ Châtelet $\grave{e}<\bar{e}[+$ n, fin. - ana, etc.

Le caractère nasal ou dénasalisé du timbre est évidemm ${ }^{\mathrm{t}}$ difficile à saisir dans des graphies influencées par l'orthogr. française. Ainsi, la c. 97 VEINE $(<\overline{\mathrm{e}}[+n)$ confond Fleurus et Châtelet sous la double notation win.ne / wêne. Par contre, les c. 69 PEINE (même base) et 90 SEMAINE (<- ana) distinguent les deux pts: Fleurus pwin.ne, samwin.ne $\sim$ Châtelet pwène, samwène. La c. SEMAINE range Farc. du côté de Châtelet.

Farc. hésite, manifestement. Guyaux donne les deux types ${ }^{\circ}$ pwène, ${ }^{\circ}$ pwin-ne (Gs 7, 9). Parfois, les f. de Malburny suivent l'ALW et le type Châtelet: ' 'pwènes 'peines' rimant avec ${ }^{\circ}$ quinzènes 'quinzaines' ( $\left.\mathrm{p} 17\right)$. Parfois, il nasalise : 'quinzainne (n 3), 'moînner 'mener' (p 17), 'minme 'même' (q 21). Modave (m 19 ; v. aussi f 5, m 13) fait pencher la balance du côté du type Fleurus, avec voy. nasale, quand il fait rimer ${ }^{\circ}$ poinnes 'peines' et 'continne 'contente' : v. reprod. 25.

\section{D.1.4. Fleurus $-e ́ z$, , $-\hat{\imath} z \sim$ Châtelet $-\grave{e} z$ à l'impér. présent, $2^{\text {ème }}$ pers. plur.}

On a vu (A.3) que les finales de la $2^{\text {ème }}$ pers. du plur., à l'ind. présent, opposaient $-O z$,$\hat{o} z$, -ouz à Fleurus et Farc. et -èz à Châtelet. Les c. 98 PESEZ(-MOI) et 99 ABAISSEZ de 1'ALW 2 montrent que cette dernière localité garde à l'impér. la finale -èz, comme au s. de l'o.-w. (v. reprod. 26). Au contraire, Fleurus se range avec le n. du domaine, qui a des f. différentes selon les verbes : -éz (PESEZ), - $\hat{\imath} z$ (ABAISSEZ). Il faut aussi tenir compte, dans le cas des auxiliaires, de la communauté de f. entre impér. et subj. prés. d'une part, et subj. prés. et subj. imparf. d'autre part. La c. 120 (QUE J')AIE fournit certains «types qui sont ou qui pourraient être originellement des imparfaits (fr. eusse), et d'autres en -iche, -isse, qui sont aussi bivalents » (ALW, p. 333).

« En principe, la $2^{\mathrm{e}}$ plur. de l'impér. prés. devrait coïncider partout avec la $2^{\mathrm{e}}$ plur. de l'ind. prés. » (L. Remacle) $\left({ }^{29}\right)$. L'ALW montre que Fleurus déroge à ce principe général. Au contraire, Farc. s'y conforme et se rapproche de ce fait du type qui domine dans l'extrême s. de $\mathrm{Na}$ et l'arr. de D, au s.-e. En effet : ${ }^{\circ}$ osos tout l' même 'osez tout de même » (Rg 13), ${ }^{\circ} n i$ vos dirindjoz 'ne vous dérangez pas'(Gs 14). Même finale sur un subj. imp. : ${ }^{\circ} N$ 'euchos né peu 'N'ayez pas peur' ( $\operatorname{Rg} 8)$. Par ailleurs, le plus fidèle de nos auteurs, Modave, donne pour Farc. une f. possible pour Fleurus : 'Euchî l'air d'ïesse di bos 'Ayez l'air d'être de bois' (f 14). Pour des v. appartenant à d'autres conjug., Guyaux a des f. de type Chât. : ${ }^{\circ}$ achîdèz-vous 'asseyez-vous' (s 16), 'fièz 'faites' (s 18), 'purdèz 'prenez' (s 17), etc. Mais la f. dijèz (s 21, $22,24)$ trouve aussi sa version en $-o z$ : Dijos èwou s' qui dj' trouv'rait 'Dites-moi où je trouverais' (MOt 3).

En conclusion, sur carte : a) la situation à Farc. ne peut être totalement assimilée à celle de Fleurus ou Châtelet; b) elle suggère une zone de mélange et d'indécision dont participent aussi les pts $\mathrm{Na} 44$ Mazy et $\mathrm{Na} 107$ Arsimont ; c) en raison de différences de conjugaisons, les c. PESEZ et ABAISSEZ ne peuvent représenter que de manière partielle et ambiguë toute la réalité.

\section{D.2. Formes en concurrence à Farciennes : (JE) SUIS}

\footnotetext{
${ }^{29}$ ALW 2, p. 267.
} 
D'une part, la c. 93 (JE) SUIS de l'ALW 2 relève deux f. à Farc. : sûu, type pic. dominant, et sêे, forme typique du s. de Ch, s'étendant sur les arrondissements de Thuin et Philippeville (Jamioulx, Cerfontaine, Philippeville...) $\left({ }^{30}\right)$. D'autre part, Farc. se trouve en bordure d'une vaste zone présentant une troisième forme, de Namur à Liège : so. Presque tous les auteurs ont toujours ${ }^{\circ} \operatorname{seu}(s)$, sêu(s). Malburny se singularise par un certain nombre de f. nam. : ${ }^{\circ} \mathrm{Dji} n$ ' sos né pus ritche pou ça 'Je ne suis pas plus riche pour ça' (n 11, 16, 26 affirm. ou nég.), 'dji sos habitouwé 'je suis habitué' (q 13).

\section{E. Imparfait, conditionnel}

\section{E.1. Imparfait, conditionnel : finales en -è, typiques de Farciennes}

Selon les c. 108 (IL) PASSAIT, 109 (J')ĖTAIS et 113 (J')AURAIS de l'ALW, Farc. se singularise à l'imparf. et au condit. par une flexion uniforme en $-\grave{e}$, au centre d'une zone où se rencontrent deux autres types généraux: d'une part -éve $<-a b a m$ au n., particulièrement à Fleurus, et vers $\mathrm{Na}$, où le type prend la forme -ê̂ve; d'autre part -êu, -eu< -ēbam. à l'o. et au s., particulièrement à Châtelet $\left({ }^{31}\right)$. La carte $\mathrm{X}$ établie par Grignard confirme que ces trois types se rencontrent particulièrement au n.-e. de Charleroi, mais elle propose une distribution partiellement différente des $\mathrm{f}$. et inscrit Farc. dans la zone $-e \hat{u}$, -eu $\left({ }^{32}\right)$. En somme, selon Grignard, le type Châtel. en -êu,-eu gagnerait davantage vers le n., aux dépens d'une zone en - è néanmoins plus consistante et homogène que dans l'ALW.

Les textes donnent plutôt raison à celui-ci en ce qui concerne Farc., malgré la précision et le soin caractérisant l'enquête de Grignard. La plupart de nos auteurs - en dehors de Malburny - reproduisent fidèlement la forme en -è : ' estais, -ait (Gs 15-23, MOf 4-10, MOm 2), 'avait (Gs 6, 12-13, etc., MOf 4 ; Rg 9), 'vayait 'voyait' (MOf 4, 5), 'fèyais 'faisais' (Gs 14), ${ }^{\circ}$ sèrait (Gs 11, 12, 24 ; MOf 4-6, 11), ${ }^{\circ}$ côperait (MOf 6), ${ }^{\circ}$ duvrais, du- (Gs 24, MOp 11), ${ }^{\circ} r$ 'lètch 'rait 'relècherait' ( $\left.\operatorname{Rg} 8,9\right)$, 'sârait (MOf 5), 'sintirait (MOf 5), vairais 'viendrait' (MOm 3), 'vôrais, vaurait, vourrait 'voudrais, voudrait' (Gs 24, MOf 4-5, MOm 2 ; Rg 9), etc.

La longueur relative de la désinence pose éventuellement un problème. La c. AURAIS de

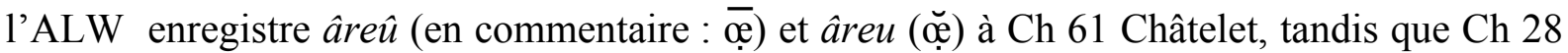
Viesville et Ch 43 Jumet, peu éloignées de Farc., ont la seule f. brève. Les graphies de nos textes sont à cet égard ambiguës. Dans l'incertitude, on a ci-dessous mentionné soit les deux f. possibles, soit la graphie $\left({ }^{\circ}-e u\right)$, soit le seul timbre $(-e u)$, notamment pour faciliter la lecture de certains tableaux.

\section{E.2. Les finales en concurrence chez Malburny}

A propos des f. de l'imp. et du cond., Grignard ajoutait : " Ce qui vient compliquer cette question de géographie linguistique, c'est que la plupart des régions utilisent deux et même trois formes d'imparfait. Dans le dom. namurois de -éve, -è sert de seconde forme, et l'euphonie seule semble en régler l'emploi; aux environs de Namur, -êuve est la forme

\footnotetext{
${ }^{30}$ La f. seû est notée pour Farc. par MARÉCHAL.

${ }^{31}$ On ne retiendra dans ce qui suit que la forme longue, la plus proche de Farc., bien que Ch 43 Jumet ait la f. brève.

${ }^{32}$ Farc. occupe une des pointes du triangle formé par Lambusart (-è ; pas de pt, même proche, à l'ALW),

Moignelée (-éve ; id. ; f. de Fleurus).
} 
longue, -eù, -è la forme brève », etc. $\left({ }^{33}\right)$ Malburny va même plus loin puisqu'il use pour le condit. des trois types généraux définis plus haut, comme l'illustre ce passage:

'quice qui m' sognerait, ci çà n' sèreu nin mi: ci n' sèrait né Colas, ni Twène, ni Bartole; dji crwè qui (...) dj'areuve waiti à çà pou mi p'tite santé, ah! yayaïe, dji n' viqureuve pus... 'qui me soignerait si ce ne serait pas moi : ce ne serait pas Colas, ni Toine, ni Bartole; je crois que (...) j’aurais veillé à ça pour ma petite santé, ah ! yayaïe, je ne vivrais plus...'(n 3$)$.

${ }^{33}$ GRIGNARD, p. 97 (471). 


\section{E.2.2.1. Classement général des formes chez Malburny}

Maréchal livrait ici, concernant le centre-w., l'observation suivante. Dans les régions de Namur et de Dinant, là où deux f. sont en concurrence $\left({ }^{34}\right)$, « une longue et une courte », c.-àd. -éve, -êuve en concurrence avec $-\grave{e},-e u$, $-e \hat{u}$, le choix peut s'appliquer à tous les types de verbes, comme à Namur et environs (Flawinne, Profondeville), ou être réservé à certains types. Il avance, dans le second cas de figure, une hypothèse : "il semblerait que la forme courte fût primitivement, ici comme à Liège, réservée à l'imparfait des auxiliaires et au conditionnel (...) de toutes les conjugaisons » $\left({ }^{35}\right)$. L'évolution aurait surtout affecté le conditionnel, dans le sens où « les patois actuels, dans une forte proportion, lui attribuent les terminaisons longues » $\left({ }^{36}\right)$. Ce n'est, en tout état de cause, pas le cas chez Malburny, où celles-ci apparaissent réservées aux auxiliaires, de façon plus nette à ce mode qu'à l'imparfait.

\begin{tabular}{|c|c|c|c|c|}
\hline \multicolumn{5}{|c|}{ Ind. imparfait } \\
\hline & Pers. & ${ }^{\circ}-\grave{e}$ & ${ }^{\circ}-e u,{ }^{\circ}-\mathrm{ou}$ & ${ }^{\circ}$-ève \\
\hline être & $\begin{array}{l}1^{\text {ère }} \text { pers. } \\
2^{\text {ème }} \text { pers. } \\
3^{\text {ème }} \text { pers. } \\
\text { impers. }\end{array}$ & $\begin{array}{c}\text { (dj') estais }(3 \mathrm{x}) \\
- \\
\text { (il) astait }(2 \mathrm{x}), \text { estait }(-y) \\
\left(c^{\prime}\right) \text { estait }(2 \mathrm{x})\end{array}$ & $\begin{array}{c}- \\
- \\
\text { (il) asteu } \\
\text { (ç') asteu }\end{array}$ & $\begin{array}{c}- \\
\text { - } \\
\text { (il) estève } \\
-\end{array}$ \\
\hline avoir & $\begin{array}{l}1^{\text {ère }} \text { pers. } \\
2^{\text {ème }} \text { pers. } \\
3^{\text {ème }} \text { pers } \\
\text { impers. }\end{array}$ & $\begin{array}{c}\text { (dj)'avais }(3 \mathrm{x}) \\
\text { (t') avais } \\
\text { (il) avait }\end{array}$ & $\begin{array}{c}- \\
- \\
\text { (il) aveut }(2 \mathrm{x}) \\
\text { (d') aveu, (gn-) aveu 'il y } \sim(2 \mathrm{x})\end{array}$ & $\begin{array}{l}- \\
- \\
- \\
-\end{array}$ \\
\hline savoir & & savais 'savais' & saveu 'savais' & \\
\hline \multirow[t]{2}{*}{ autres } & & & $\begin{array}{c}\text { alleu 'allais' } \\
\text { chèneu 'semblait' } \\
\text { d'vout 'devait' }\end{array}$ & \\
\hline & & $\begin{array}{c}\text { dijais 'disais' } \\
\text { feyais, fiais 'faisais' } \\
\text { djouais 'jouais' } \\
\text { daurmais 'dormais' } \\
\text { foutais 'foutais' } \\
\text { thais 'tenais' }\end{array}$ & & \\
\hline
\end{tabular}

\footnotetext{
${ }^{34}$ Qqs villages emploient exclusivement -eù (au s. de Na 1: Crupet, Lustin, Yvoir, Sosoye) ou -⿳亠丷 (au s.-o. : canton de Roux-Biesme).

${ }^{35}$ Cette situation « primitive » se conserverait dans la région de Jallet, ou Na '103 Perwez (pt régulier proche : Na 101 Ohey), au s.-e. d'Andenne.

${ }^{36}$ MARÉCHAL 1900, p. 79.
} 


\begin{tabular}{|c|c|c|c|}
\hline \multicolumn{4}{|c|}{ Conditionnel prés. } \\
\hline Pers. sing. & ${ }^{\circ}-\grave{e}$ & ${ }^{\circ}-e u$ & ${ }^{\circ}$-euf, ${ }^{\circ}$-euve \\
\hline $\begin{array}{l}1^{\text {ère }} \text { pers. } \\
2^{\text {ème }} \text { pers. } \\
3^{\text {ème }} \text { pers. } \\
\text { impers. }\end{array}$ & $\begin{array}{c}- \\
- \\
\text { sèrait }(-y) \\
(\text { ci } n \text { ') sèrait }(2 \mathrm{x})\end{array}$ & $\begin{array}{c}- \\
- \\
- \\
\left(c ̧ a n^{\prime}\right) \operatorname{sereu}(2 \mathrm{x})\end{array}$ & $\begin{array}{c}\text { (dj') sèreuf } \\
- \\
- \\
-\end{array}$ \\
\hline $\begin{array}{l}1_{\text {ère }}^{\text {èrers. }} \text { pers. } \\
2^{\text {ème }} \text { pers. } \\
3^{\text {ème }} \text { pers. } \\
\text { impers. }\end{array}$ & $\begin{array}{c}\text { (dji n') arais } \\
- \\
\text { s'arait(-i) } \\
-\end{array}$ & $\begin{array}{l}- \\
- \\
- \\
-\end{array}$ & $\begin{array}{c}\text { (dj') arreuve }(2 \mathrm{x}) \\
- \\
- \\
-\end{array}$ \\
\hline & & sareu 'saurais' $(2 \mathrm{x})$ & \\
\hline & faurait 'faudrait' & faureu 'faudrait' & \\
\hline & & $\begin{array}{c}\text { rireut 'rirait' } \\
\text { vaureu 'voudrait' }\end{array}$ & viqureuve 'vivrait' \\
\hline & $\begin{array}{l}\text { dirais, - } t \text { 'dirais, -t' } \\
\text { frais, - } t \text { 'ferait' }(2 \mathrm{x}) \\
\text { craindrais 'craindrait' } \\
d \text { 'vairais 'deviendrait' } \\
\text { daurait 'donnerait' } \\
\text { lairais 'laisserais' }(2 \mathrm{x}) \\
\text { payerais, - } t \text { 'paierais, -t' } \\
\text { préférerais 'préférerais' } \\
\text { rachetrais 'rachèterais' } \\
\text { spirierais 'briserais' } \\
\text { sognerait 'soignerait' } \\
\text { tchairait 'tomberait' } \\
\text { tolèr'rais 'tolérerais' } \\
\text { vairait 'viendrait' }\end{array}$ & & \\
\hline
\end{tabular}

Malburny, par les f. en ${ }^{\circ}$-eut, ${ }^{\circ}$-éve, ${ }^{\circ}$-euve, subit sans doute l'attraction des régions voisines. Les $\mathrm{f}$. en $-\grave{e}=-$ ais, qui pourraient sembler modernes par rapport aux précédentes, seraient en réalité archaïques ou traditionnelles, auquel cas elles appartiendraient éventuellement à une ligne de désinences «spontanées » ou «naturelles » $\left({ }^{37}\right)$ dont la c. PASSAIT dessine le tracé (de AR[denne] 1 Givet à Nivelles 39) de manière d'autant plus significative que la probabilité de trouver $-\grave{e}$ est moindre dans un v. de la $1^{\text {ère }}$ conjug. $(<-$ abam) .

Dans les f. courtes, le choix de ${ }^{\circ}-$ eut, ${ }^{\circ}$-out affecte surtout, à l'imp. comme au condit., des v. susceptibles d'une construction avec infinitif, qui remplissent donc une fonction de semi-auxiliaire : savoir, vouloir, devoir, falloir, aller (fut. inchoatif). L'alignement de savoir sur avoir est connu (ALW 2, 299a ; GRIGNARD 471); il donne ici lieu au seul exemple de f. en ${ }^{\circ}-e u$ à l'imp. et au condit . pour un même non-auxil. Dans les f. longues provenant du lat. -abam, -abat, celles en -ève/-éve semblent réservées à l'imp. d'être, celles en -êuve au cond. d'être ou avoir.

Notons encore :

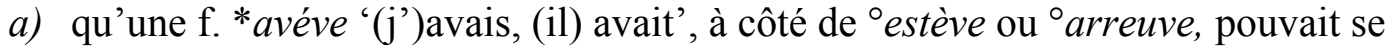
disqualifier par répétition déplaisante $\mathrm{du}-v$ - (prévention dissimilative).

b) que les f. en ${ }^{\circ}-e u$ apparaissent exclusivement à la $3^{\text {ème }}$ pers. de l'imparf. et particulièrement avec sujet impers., pour être et avoir, tandis que les $\mathrm{f}$. en ${ }^{\circ}$-euf, -euve dominent à la $1^{\text {ère }}$ pers. au cond.

\footnotetext{
${ }^{37}$ REMACLE, commentaire de PASSAIT confirmant MARĖCHAL, p. 77, n. 2.
} 
Maréchal ajoute en conclusion qu'à certains endroits, on combine volontiers deux types en présence pour des raisons d'harmonie : i faurè qu' plourève 'il faudrait qu'il pleuvrait' (SartSaint-Laurent), si djel veyè, djel riconiréve bé 'si je le voyais, je le reconnaîtrais bien'(Vitrival). Ailleurs, on préférera la symétrie : Si djel vièrò, djel riconèrò 'si je le verrais, je le reconnaîtrais' (Liernu). Ceci nous engage à considérer la sélection des f. à choix dans divers types de corrélations.

\section{E.2.2.2. Distribution en proposition principale + conditionnelle chez Malburny}

\begin{tabular}{|c|c|}
\hline \multicolumn{2}{|r|}{$S i+$ v. subord. + v. principal } \\
\hline $\begin{array}{l}S i+\text { imparf. }+ \\
\text { condit. }\end{array}$ & \\
\hline \multirow[t]{2}{*}{$-e u+-\grave{e}$} & $\begin{array}{l}\text { 1) }{ }^{\circ} \text { Ci n' sèrait co ré si ç'asteu l' prumî côp ... 'ce ne serait encore rien si } \\
\text { c'était la première fois ...' (q 7) }\end{array}$ \\
\hline & $\begin{array}{l}\text { 2) 'S'il aveut co d'mèrè ci pu longtimps, dji n'arais pu pon yeu d' } \\
\text { pratiques 'S'il était (avait) resté ici plus longtemps, je n'aurais plus eu de } \\
\text { pratiques' (q 10) }\end{array}$ \\
\hline$-\grave{e}+-e u f$ & $\begin{array}{l}\text { 3) 'Si dj’ vos djouais in air, patron, est-ce qui dj’ sèreuf quitte ?‘Si je vos } \\
\text { jouais un air, patron, serais-je quitte?' (n 11) }\end{array}$ \\
\hline$-\grave{e}+-\grave{e}$ & $\begin{array}{l}\text { 4) }{ }^{\circ} \text { S'il astait din l' gayolle, on dirait...'S'il était dans la cage, on } \\
\text { dirait...'(p 18) } \\
\text { 5) }{ }^{\circ} \text { si dji t'nais l' vauré (...), dje l' sipierais... 'si je tenais le vaurien (...), } \\
\text { je le mettrais en miettes' (p 25) }\end{array}$ \\
\hline \multicolumn{2}{|l|}{$\begin{array}{l}S i+\text { condit. }+ \\
\text { condit. }\end{array}$} \\
\hline${ }^{\circ}-e u+-\grave{e}$ & $\begin{array}{l}\text { 6) 'quice qui m' sognerait, ci çà n' sèreu nin mi 'qui me soignerait si ce ne } \\
\text { était (serait) pas moi' '(n 3) }\end{array}$ \\
\hline${ }^{\circ}$-euve + -è & $\begin{array}{l}\text { 7) 'sinon qui dj'arreuve trop peu d'vos embaiter, dji vos ès dirais cô bé } \\
\text { d's'autes 'sinon que j'aurais trop peur de vous embêter, je vous en dirais } \\
\text { encore bien d'autres' (n 4). }\end{array}$ \\
\hline$-\grave{e}+-\grave{e}$ & $\begin{array}{l}8)^{\circ} \mathrm{Ci} \text { n' sèrait ré, si au d'bout des djous di crèdit (...), çà tchairait 'Ce ne } \\
\text { serait encore rien si, au début des jours de crédit }(\ldots) \text {, ça tomberait' }(\mathrm{n} 3) \text {. }\end{array}$ \\
\hline
\end{tabular}

Le type fr. [imp. + cond.] peut être considéré comme dominant. Quand ÊTRE et AVOIR figurent à la fois en princ. et en subord., l'auteur choisit des finales différenciant les v. par le timbre, dans une distribution de type $s i+$ imp. $-e u+$ condit. $-\grave{e}:$ ex. 1-2. Quand la corrélation fait intervenir un auxiliaire, la différenciation des types de v. peut être renforcée par celle des désinences (ex. 3) ou estimée suffisante et s'accommoder dès lors de leur symétrie (ex. 4). Celle-ci est assurée quand les v. non-auxil. sont différents : ex. 5.

Le même type de « règle » s'applique aux conditionnels corrélés. Les ex. 6-7 sont analogues à l'ex. 3 . On remarquera que les ex. 3 et 7 réservent la $\mathrm{f}$. longue à la $1^{\text {ère }}$ pers. du sing. L'ex. 8 est analogue à l'ex. $4:$ il admet la même désinence quand les v. sont de type différent. 


\section{E.2.2.3. Distribution en proposition principale + subord. COD chez Malburny}

Considérons ensuite les f. choisies dans un deuxième type de distribution.

\begin{tabular}{|c|l|}
\hline$-e u+-\grave{e}$ & $9)^{\circ}$ Dji saveu bé qui dj'ès daurais 'Je savais bien que j'en aurais' (n 5) \\
\hline$-\grave{e}+-\grave{e} v e$ & $\begin{array}{l}\text { 10) }{ }^{\circ} \text { Dj'ès l' dijais bé qui l' patron estève mau tourné 'Je le disais bien } \\
\text { que le patron avait (était) mal tourné' (n 10) }\end{array}$ \\
\hline$-e u+-\grave{e}$ & $\begin{array}{l}\text { 11) }{ }^{\circ} \text { dj'alleu djustumint ti d'monder si ti fiais li martchand d' canadas ! } \\
\text { 'j'allais justement te demander si tu faisais le marchand de pommes de } \\
\text { terre !' (p 9/Zi) }\end{array}$ \\
\hline$-\grave{e}+-\grave{e}$ & $\begin{array}{l}\text { 12) }{ }^{\circ} \text { qwont dji t'ès dijais qui ti d'vairais ritche in côp! 'quand je te le } \\
\text { disais que tu deviendrais riche d'un coup' (Ne 23) }\end{array}$ \\
\hline$-e u+-e u$ & $\begin{array}{l}\text { 13) }{ }^{\circ} \text { Im' chèneu bé qu'il asteu ici 'Il me semblait bien qu'il était ici' (n } \\
\text { 8bis) }\end{array}$ \\
\hline
\end{tabular}

Application du même principe. La différenciation est prise en charge par la désinence dans la corrélation de deux auxiliaires ou semi-auxil. (ex. 9, avec savoir fonctionnant comme avoir et être). Elle est renforcée par la dés. dans la corrélation d'un auxil. ou semi-auxil. assimilé ( ${ }^{\circ}$ alleu) et d'un v. de type différent, quel que soit l'ordre de distribution (ex. 10-11). Elle est prise en charge par la différence des v., qui prennent le même dés. en Farc. aux non-auxil. (ex. 12). On n'a pas vu de raison expliquant l'ex. 13 - qui s'oppose au principe générique. Euphonie ?

Dans les propositions indépendantes, être et avoir prennent presque toujours la f. $-\grave{e}$ de Farc. à l'imparf. et au condit. Ceci vaut notam ${ }^{t}$ pour la $1^{\text {ere }}$ pers. du sing. $\left({ }^{38}\right)$. Les f. en $-e \hat{u}$, $e u$ sous influence du s. apparaissent presque exclusivem ${ }^{t}$ avec impersonnel $\left({ }^{39}\right)$. Ceci s'étend en partie aux v . que nous avons considérés comme semi-auxil. : ${ }^{\circ}$ Et audjourdu lindi, y faureu ptète lèŷ̂ mi tchimige 'Et aujourd'hui lundi, il faudrait peut-être laisser ma chemise' (n 11), 'Dins l' commerce, si on s'vaureu fer des biles! 'Dans le commerce, si on voudrait se faire de la bile !' (n 3).

Proposons pour terminer le système matriciel suivant $\left({ }^{40}\right)$.

\footnotetext{
38 ○Min dj'estais si bé mwé après m' garçon coëffeu 'Mais j’étais si fâché après mon garçon coiffeur' (q 10), 'Té, dj'estais co bé èsdaurmi! 'Tiens, j'étais encore bien endormi' (q 12), 'dj'avais bé rouvi li cène dou flamind 'j'avais bien oublié la scène du Flamand' (p 27), `dj'avais compris in boc 'j'avais compris un bock' (q 12), 'Si dj'estais vo pére, garnement... 'Si j'étais votre père, garnement...' ( $\mathrm{p} \mathrm{17),}{ }^{\circ}$ sèrait-y complice ? 'serait-il complice' (q 17), 'In crime s'ârait-i commis droci ! 'Un crime se serait-il (aurait) commis ici !' (q 12), etc. $39 \circ$ il esteut timps ' il était temps' (q 18), 'Y gn'aveut deux 'Il y en avait deux' (q 19), 'Dou momint qui gn-aveu qu' moi qu'aveu pinsé à ça 'Du moment qu'il n'y avait que moi qui avais pensé à ça' (p 20)

${ }^{40}$ L'exemple ci-dessous, qui comporte deux impers., semble contredire la symétrie de l'ex. 9 : ${ }^{\circ}$ si vairais ène saqui... ça n' sereu né convenâpe 's'il venait quelqu'un... ce ne serait pas convenable' (n16). Le contexte montre qu'il ne s'agit qu'en apparence d'une corrélation avec subord. conditionnelle : ${ }^{\circ}$ Passe mi les vigeries... et hardant là pace qui si vairais ène saqui... ça n' sereu né convenâpe di vir in champête su ène tchèyère, là c'est fait 'Passe-moi les vieilleries... et vite, parce que s'il venait quelqu'un... ce ne serait pas convenable de voir un garde-champêtre sur une chaise, là c'est fait'.
} 


\begin{tabular}{|c|c|c|c|}
\hline auxil. + auxil. & \multicolumn{2}{|c|}{ eu (imparf.) + è (cond.) } & $\begin{array}{c}\text { si ç'asteu + i n'sèrait (1) } \\
\text { s'il aveut + dji n'arais (2) } \\
\text { dji saveu + dj'ès daurais (10) }\end{array}$ \\
\hline \multirow[t]{4}{*}{$\begin{array}{l}\text { auxil. + non- } \\
\text { auxil. }\end{array}$} & \multirow[t]{2}{*}{$\begin{array}{l}\text { dissymétrie } \\
\text { renforcée } \\
\text { par désinences }\end{array}$} & $\begin{array}{l}\text { auxil. princip }{ }^{\mathrm{t}} 1^{\text {ère }} \text { pers. } \\
+ \text { non-auxil. } \\
\text { euve, éve }+ \text { è }\end{array}$ & $\begin{array}{c}\text { dj' sèreuf }+ \text { dj' vos djouais (3) } \\
\text { dj'arreuve }+ \text { dji dirais }(8) \\
\text { (il) estève }+ \text { dj'ès l' dijais }(11)\end{array}$ \\
\hline & & $\begin{array}{c}\text { aux. } 3^{\text {ème }} \text { impers. } \\
+ \text { non-auxil. non-impers. } \\
\text { eu }+ \text { è }\end{array}$ & ça n' sèreu + sognerait (6) \\
\hline & \multirow[t]{2}{*}{ symétrie } & $\begin{array}{c}\text { auxil. } 3^{\text {ème }} \\
+ \text { non-auxil. impers. } \\
\text { è }+ \text { è }\end{array}$ & $\begin{array}{c}\text { ci n' sèrait + ça tchairait (9) } \\
\text { s'il astait + on dirait (4) }\end{array}$ \\
\hline & & $?$ & chéneu / asteu (13) \\
\hline $\begin{array}{c}\text { non-auxil. }+ \\
\text { non-auxil. }\end{array}$ & \multicolumn{2}{|r|}{ è̀ $+\grave{e ̀}$} & $\begin{array}{l}\text { t'nais / sipierais (5) } \\
\text { dijais / vairais (14) }\end{array}$ \\
\hline
\end{tabular}

\section{H. CONCLUSION}

Arrêtons-là un examen qui pourrait être étendu à d'autres traits phonétiques ou morphologiques, sur la seule base des Atlas linguistiques de la Wallonie 1 et 2. Ainsi, les textes font parfois apparaître une f. pronominale étrangère à Farciennes ou, au contraire, en ignorent une autre suggérée par la carte. Selon la c. 38 LEUR (devant le verbe et devant consonne) de l'ALW 2, tout l'arrondissement de Charleroi a lyeû, notamment à Fleurus et Châtelet, et lêu, f. enregistrée à Farc. Malburny emploie celle-ci, mais aussi l'zeû, qui est de type namurois et en usage, il est vrai, dans une localité voisine (Na 107 Arsimont) : ${ }^{\circ} D j i n$ ' pou né pourtant l'z'eu r'fuser à boëre 'Je ne peux pourtant pas leur refuser à boire' $(N 4),{ }^{\circ} D j$ ' va l'zeu dire 'Je vais leur dire', 'Dji l'zeu donne 'Je leur donne' $(N 16)$. D'autre part, la c. 8 $\mathrm{UN}$ (nominal) mentionne yink pour une zone étroite couvrant l'axe Ch 33-61. Cette f. n'apparaît pas dans les textes vus, qui ont 'yin 'un' (Rg 4), 'yen (MAn 4, MAp 4, 12, 13, MAq 4, 13), ${ }^{\circ}$ ien (MOs 2, 13), `yun (MAp 12, 18) ${ }^{41}$.

Maigres résultats, dira-t-on en conclusion, pour une si longue enquête. Les textes confirment le rôle de la ligne Fleurus-Farciennes-Châtelet en tant que

a) limite extrême du domaine wall. :

- ou, ôे < ั̆[, c. BCEUF, (JE) VEUX $={ }^{\circ}$ voux, ${ }^{\circ}$ voûx $[\mathrm{C} .1]$

$-w a, w \hat{a}<\breve{\mathrm{o}}]+\mathrm{r}$, c. PORTER $={ }^{\circ}$ pwoirter $[\mathrm{C} .2]$

b) limite extrême du domaine nam. pour le part. passé fém. en -euwe [A.2]

Quand les c. font apparaître une distinction entre Ch 33 Fleurus au n. de Farciennes et Ch 61 Châtelet au s., c'est généralement sur Fleurus que s'aligne la f. de Farc., notamment en matière de maintien des voy. nasales:

$$
-\grave{e}<\overline{\mathrm{e}}+\mathrm{y}, \text { c. } 45 \text { FROID }=\text { nam. }{ }^{\circ} \text { frèd , frêd [D.1.1] }
$$

\footnotetext{
${ }^{41}$ Par contre, Malburny a ${ }^{\circ}$ li quinque 'lequel' (p 16), qui correspond à une f. nasalisée ou semi-nas. localisée dans le n. de l'arr. de $\mathrm{Na}$ et dans l'e. de Ni, (li) kink, $k e^{n} k$, alors que la f. de Ch 33-61 est dénas. (kék). V. aussi ol'minque 'la mienne' (p. 25).
} 
- an sans vélarisation en $\stackrel{a}{n}$, c. 9 CHAMBRE = tchambe [D.1.2]

- hésitation entre in et $\dot{e}<\overline{\mathrm{e}}[+$ n, c. 69 PEINE, mais la voy. nasalisée semble plus authentique $=$ pwin-ne, poinnes [D.1.3]

Dans les cas D.1.1 et D.1.3, Farc. appartient au domaine nam. ou domaine proprem ${ }^{t}$ wall. (qui nasalise plus généralement), lequel apparaît plus étendu que ne le suggèrent les c. Ceci vaut aussi pour :

- La désinence nam. en $-o z$, $-\hat{o} z$ de la $2^{\text {ème }}$ pers. du plur. de l'indic. présent domine, malgré une certaine attraction du type de Châtelet en -ouz et du type pic. et o.-w. en -èz [A.3]. La ligne d'isoglosse entre w. et pic./o.w. se situait-elle autrefois plus au sud, au delà de Ch 61 Châtelet, entre cette localité et Ch 72 Gerpinnes, comme dans la c. 101 VOULEZ(-VOUS)?

- Les textes vérifient aussi l'appartenance de Farc. au type nam. en -roz de la $2^{\text {ème }}$ pers. du plur. du futur, conformément à la c. 107 (VOUS) AUREZ de l'ALW [A.4].

- Ils montrent également, pour la $2^{\text {ème }}$ pers. du plur. de l'impératif, une prédominance ou survivance de la f. nam. en $-O z$, que ne laissent en rien supposer les c.. 98 PESEZ(-MOI) et 99 ABAISSEZ de l'ALW 2 [D.1.4]. La situation à Farc. ne peut être totalement assimilée à celle de Fleurus ou Châtelet, et elle suggère une zone de mélange et d'indécision s'étendant vers $\mathrm{Na} 44$ Mazy et $\mathrm{Na} 107$ Arsimont.

Comme dans les cas précédents, Farc. appartient plutôt au domaine wallon en ce qui concerne la f. dominante pour (JE) SUIS : la localité privilégie la $\mathrm{f}$. de l'o.-W. sur la f. pic.

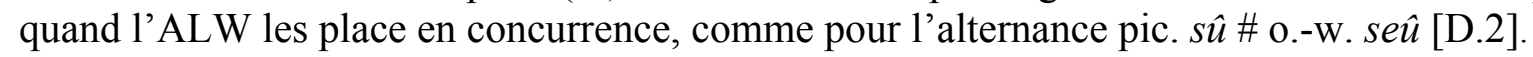

On serait tenté de conclure que Farc. s'avère principalement wallonne. Mais elle est franchement picarde, dans les cas suivants :

- ou< $<$ ó $]$, c. CROÛTE $={ }^{\circ}$ crousse $[$ C.3]

$-\left(\mathrm{J}^{\prime}\right) \mathrm{AI}=\dot{e}$, , (JE) DIRAI $=-\dot{e},(\mathrm{IL}) \mathrm{SERA}=-a[\mathrm{~B} .1]$

Si Farc. balance dès lors entre les deux régimes, elle montre aussi un caractère particulier bien affirmé, un «non-alignement » sur pic. et wallon, dans les cas suivants :

- en matière de traitement du é $[$, les textes montreraient plutôt l'extension d'un type o.w. en wè, spécifique, distinct des f. en wa qui s'étendent de part et d'autre du domaine, vers Namur ou vers Mons, Soignies, etc. [A.1].

- ses imparfaits et conditionnels en -è échappent aux clivages entre variétés régionales et ne constituent p.-ê. pas davantage une éruption locale du français, comme on l'a suggéré à propos de Malburny [E et E.2.2.1].

L'enquête qu'on a proposée permet-elle par ailleurs de privilégier, du point de vue littéraire, les auteurs dont la langue paraît la plus homogène, c.-à-d. une pratique supposée la plus conforme à une norme elle-même supposée homogène? Dans cette perspective, Joseph Modave paraît l'écrivain le plus représentatif de Farciennes, le plus fidèle à son parler. Ernest Guyaux et Camille Malburny se montrent affectés par la tendance belgo-picarde et nivelloise à l'amuissement du $-r$ - dans crwère 'croire', dwète 'droite', etc. Guyaux subit en outre l'attraction du type pic. en $-e z$, à la $2^{\text {ème }}$ pers. du plur. de l'ind. présent et il hésite entre pwène « peine » et pwin-ne, cette dernière f. semblant la plus répandue à Farc. Malburny subit de son côté la plus forte attraction de la monophtongaison du $\breve{o}]+\mathrm{r}$ en pic. et o.-w. : èpôrter 'emporter', dôrmi 'dormir', fôrt 'fort' (w. nam. èpwarter, dwarmi, fwart). Alors que Farc. s'apparente à Fleurus en matière de désinence en $-\hat{o} z$ à la forme interrogative de la $2^{\text {ème }}$ pers.du plur. de l'ind. présent, Renson subit l'infuence de la f. de Châtelet en -ouz. 
L'examen des «écarts 》 de Malburny a ouvert l'hypothèse d'un modèle générique permettant de rendre compte du plus grand nombre de ceux-ci. Une telle matrice, organisant des données liées à la variation géographique, pourrait-elle dans d'autres cas se trouver projetée en retour du plan de l'idiolecte vers celui du diasystème?

\section{REPRODUCTIONS}

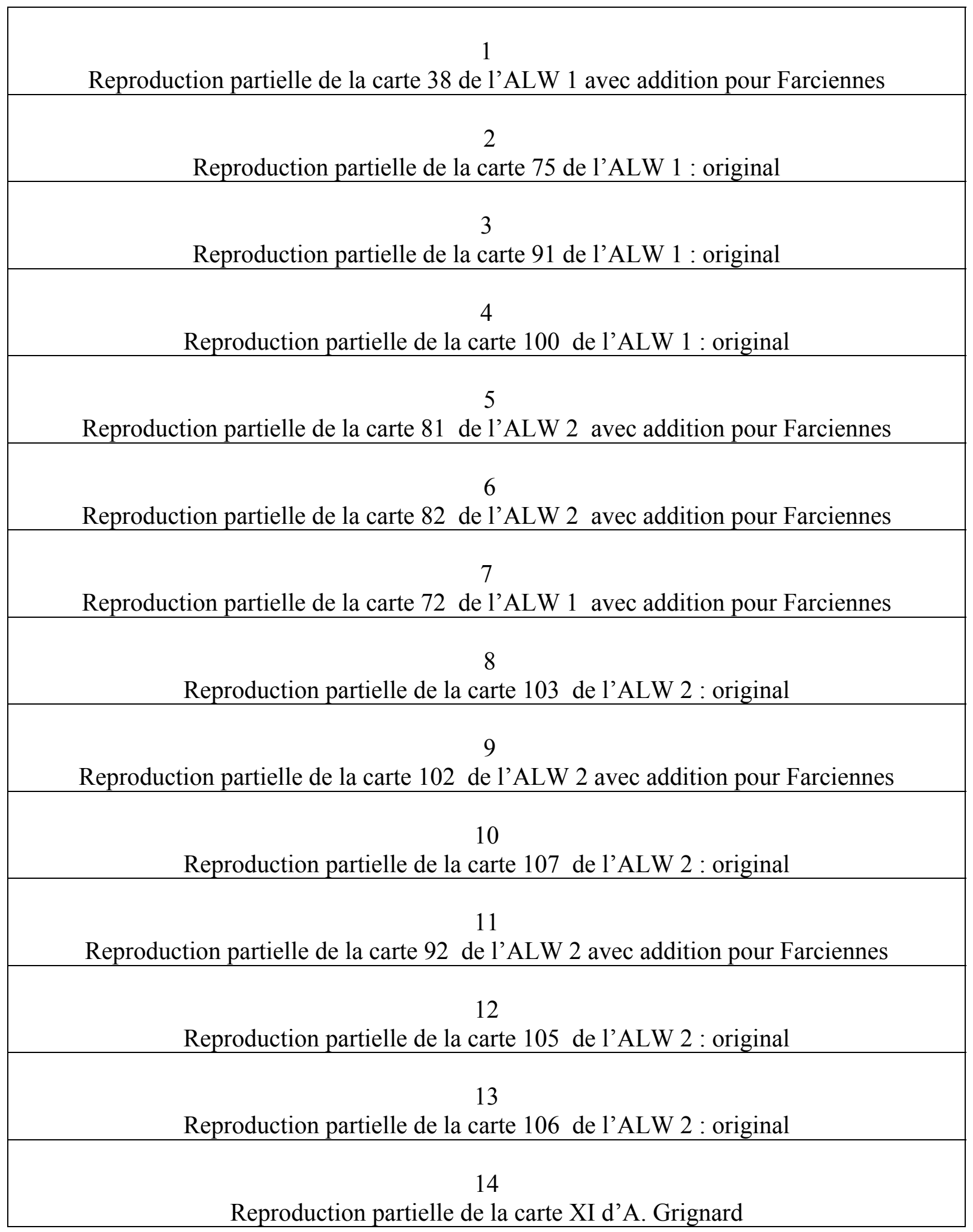


Reproduction partielle de la carte 79 de l'ALW 1 : original

16

Reproduction partielle de la carte 79 de l'ALW 1 avec addition pour Farciennes

17

Reproduction partielle de la carte 4 de l'ALW 1 avec addition pour Farciennes

18

Reproduction partielle de la carte 62 de l'ALW 1 : original

19

Reproduction partielle de la carte VI d'A. Grignard

20

Reproduction partielle de la carte XII d'A. Grignard

21

Reproduction partielle de la carte 25 de l'ALW 1 avec addition pour Farciennes

22

Reproduction partielle de la carte 45 de l'ALW 1 avec addition pour Farciennes

23

Reproduction partielle de la carte V d'A. Grignard

24

Reproduction partielle de la carte 9 de l'ALW 1 avec addition pour Farciennes

25

Reproduction partielle de la carte 69 de l'ALW 1 avec addition pour Farciennes

26

Reproduction partielle de la carte 98 de l'ALW 2 avec addition pour Farciennes 